\title{
Flora an der Chinesischen Mauer bei Jiankou
}

\author{
Eva Miller
}

\begin{abstract}
The Great Wall of China was declared as a World Heritage Site in 1986 and has been long an identity-endowing symbol of China. It runs mainly through remote areas which sometimes are difficult to access. This article deals with the vegetation of the Jiankou section of the wall near Beijing which is relatively easy to access but has not been developed for touristic purposes.
\end{abstract}

\section{Zusammenfassung}

Die Chinesische Mauer ist seit 1986 anerkanntes Weltkulturerbe und seit jeher eines der identitätsstiftenden Wahrzeichen Chinas. Sie führt in weiten Teilen durch abgelegene und bisweilen schwer zugängliche Gebiete. Dieser Artikel beschäftigt sich mit der Vegetation an und auf dem Mauerabschnitt von Jiankou in der Nähe von Peking, der verhältnismäßig gut zu erreichen, jedoch nicht touristisch erschlossen ist.

\section{Einleitung}

Die lange Mauer, die sich im Norden Chinas durch die Landschaft windet, fasziniert mit ihren schieren Ausmaßen, ihrer langen Geschichte und den wechselvollen Landschaften. Von Peking aus bequem zu erreichen, ist sie ein beliebtes Ausflugsziel und lädt zu mehr oder weniger waghalsigen Begehungen ein, getreu dem von MAO Zedong formulierten Sinnspruch: „Nur wer die Mauer besucht hat, ist ein echter Kerl.“

Die so genannte wilde, weil nicht restaurierte, Mauer von Jiankou, etwa zwei Autostunden von Peking entfernt im Norden gelegen, bietet neben zahlreichen Türmen, steilen Anstiegen und wilden Abbrüchen auch atemberaubende Ausblicke auf

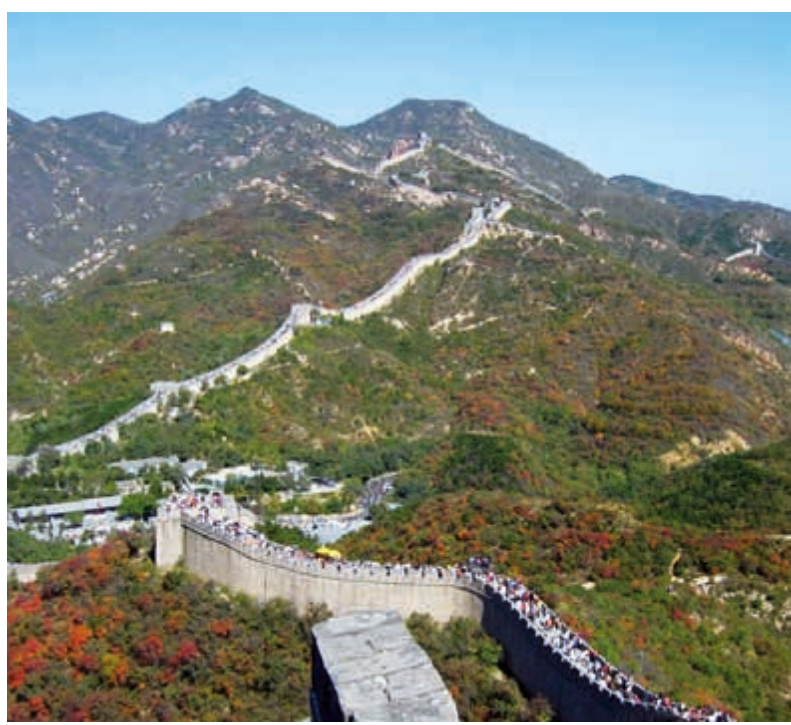

Abb. 1: Die Chinesische Mauer bei Badaling während der Oktoberfeiertage - ein Besuchermagnet. die Landschaft. So ist es nicht verwunderlich, dass der Besucher die auf und an der Mauer anzutreffende Vegetation nur am Rande wahrnimmt und ihr meist kaum Beachtung schenkt. Da die Pflanzenvielfalt aber zumindest ebenso großartig und erstaunlich ist wie die Chinesische Mauer selbst, lohnt sich ein zweiter Blick und das Umschalten von Weitwinkel- auf Makro-Objektiv durchaus.

\section{Die Chinesische Mauer}

\subsection{Lage}

Die Chinesische Mauer erstreckt sich im Norden Chinas auf einer Länge von knapp $6300 \mathrm{~km}$ von Shanhaiguan (Pass zwischen Berg und Meer) an der Küste des Golfes von Bohai im Osten bis nach

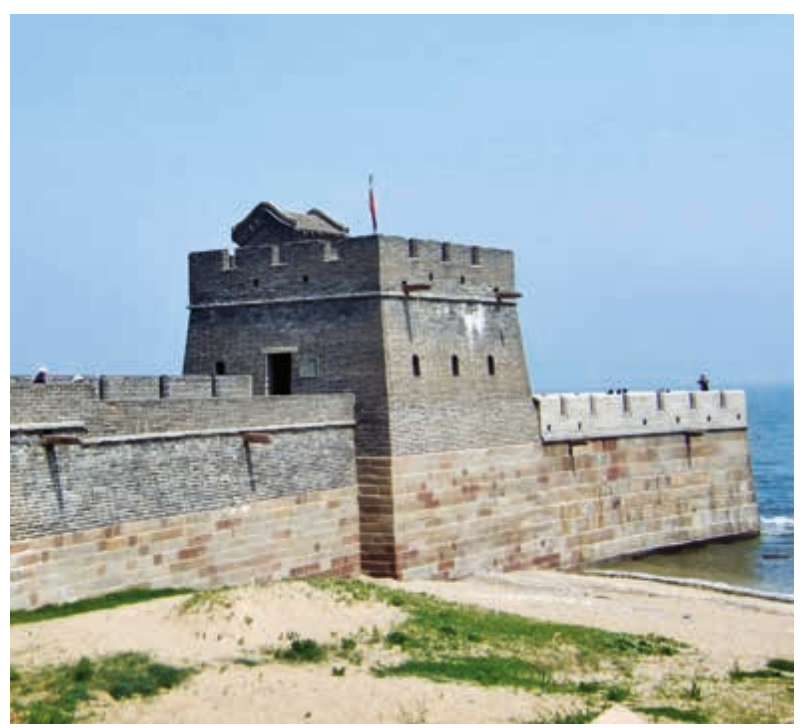

Abb. 2: Der „Kopf des Alten Drachens“ - Östlicher Endpunkt der Mauer bei Shanhaiguan am Golf von Bohai. 
Yumenguan (Jadetorpass) in der Provinz Gansu im Westen. Rechnet man, wie die offiziellen chinesischen Stellen, auch natürliche Barrieren wie Flüsse und Berge hinzu, so ergibt sich sogar eine Gesamtlänge von $8850 \mathrm{~km}$.

\subsection{Geschichte}

Bereits vor mehr als 2500 Jahren errichteten einzelne Fürstentümer in China Mauern und Wälle, um sich so vor Einfällen nördlicher Nomadenstämme zu schützen. Nach der Einigung des Reiches im Jahr 221 v. Chr. durch den ersten Kaiser, QIN SHI HuangDi, ließ dieser erstmalig eine weitläufige Gesamtanlage durch die Verbindung bestehender und den Bau neuer Abschnitte errichten. Diese Mauer diente nicht nur dem Schutz gegen die „nördlichen Barbaren“, sondern war auch Manifestation des Herrschaftsanspruches des QIN-Kaisers über die südlich der Mauer gelegenen Gebiete. Auch spätere Dynastien errichteten oder unterhielten Schutzwälle, bisweilen verfielen aber auch weite Teile der Verteidigungsanlagen, insbesondere während der Yuan-Dynastie, der von Kublai Khan (dem Enkel des Dschingis KhaN) begründeten mongolischen Herrschaft über China.

Unter dem Rebellenführer ZHU YUANZHANG wurden 1363 die mongolischen Herrscher nach 92 Jahren wieder vom Drachenthron gestürzt und aus dem Land nach Norden gedrängt. ZHU YuANZHANG selbst proklamierte sich zum ersten Kaiser der Ming-Dynastie. An den Grenzen des Reiches schuf er neue Fürstentümer und setzte dort seine Söhne als Statthalter ein. Er selbst residierte in der Hauptstadt Nanking im Zentrum des Landes. Das Fürstentum Yan im Norden des Reiches wurde vom bis dahin mongolischen Dadu aus regiert, das in Beiping (Nördlicher Friede) umbenannt worden war.

Zum Schutz der nördlichen Grenzen ließ der Hongwu-Kaiser ab1368 eine enorme Verteidigungsanlage errichten, in bewährter Manier unter Einbeziehung bestehender Bauwerke. Diese Anlage, die der Grenze zwischen nomadischer Viehwirtschaft und Ackerbau folgte, legte den Herrschaftsbereich der Ming fest und verwies die überwiegend nomadischen Mongolen und andere Reitervölker in die nördlichen Steppen- und Wüstengebiete. Allerdings stellte ihre unmittelbare Nachbarschaft für das Reich der Ming weiterhin eine ständige Bedrohung dar.

\subsection{Baubefund}

Das Aussehen der Mauer ist auf ihre Gesamtlänge betrachtet nicht einheitlich, sowohl hinsichtlich der Maße als auch bezüglich des Baumaterials, da überwiegend an Ort und Stelle vorgefundene

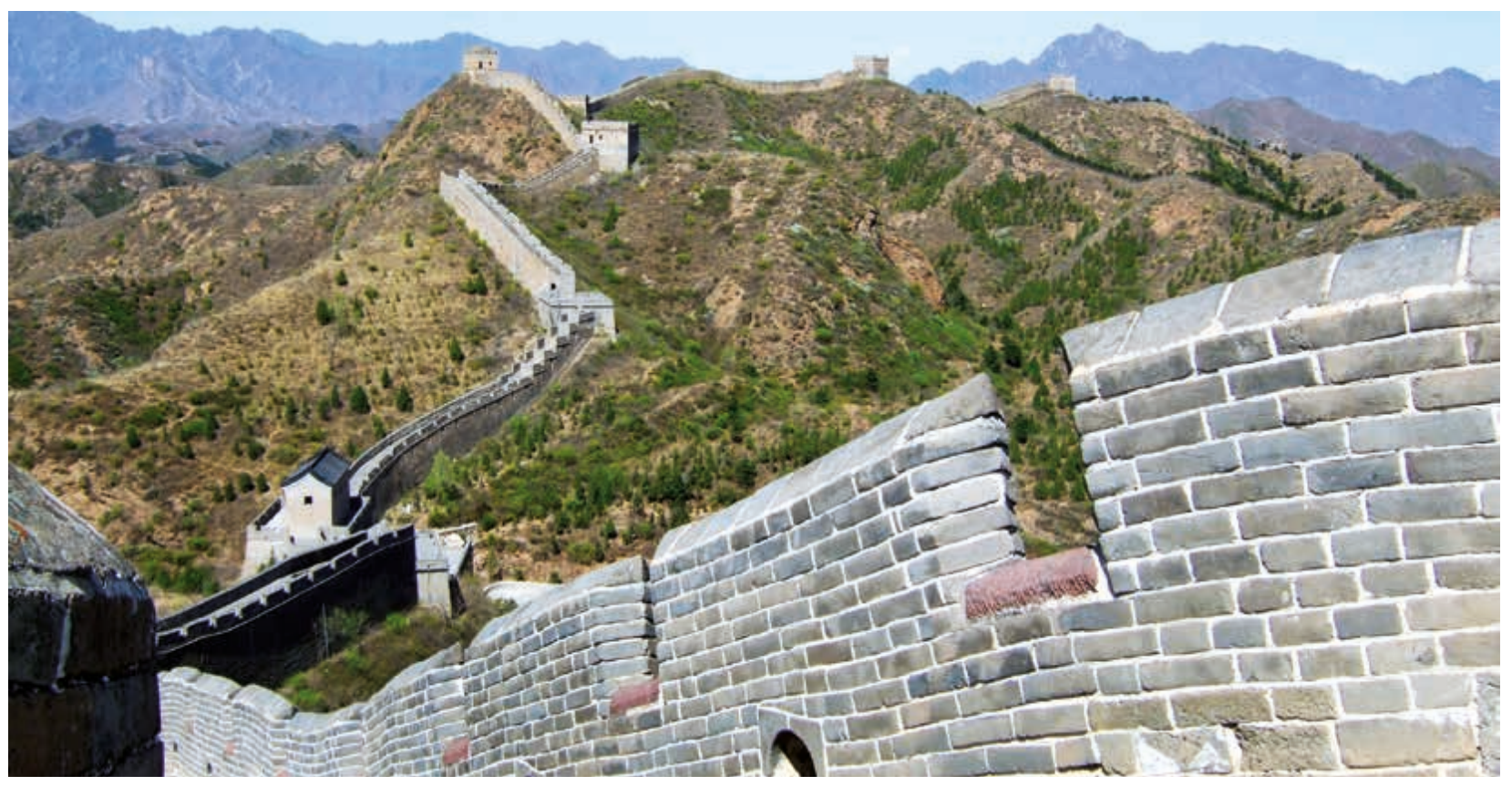

Abb. 3: Jingshanling. Der Alte Drache schlängelt sich durch die Landschaft bei Jingshanling. 
Nach dem Ende der Ming-Dynastie 1644 wurde an der großen Mauer nicht weitergebaut: Die nachfolgend herrschenden Kaiser der mandschurischen QING-Dynastie bezogen ihr angestammtes Herrschaftsgebiet in der Mandschurei sowie die Koreanische Halbinsel und das Gebiet der heutigen Mongolei in ihr neues Reich ein, die Mauer lag nun also inmitten des chinesischen Reiches. Außerdem war die Bedrohung durch "nördliche Barbaren“ dadurch gebannt, dass diese selbst auf dem Drachenthron saßen. Auch nach dem Ende des Kaiserreiches 1911 bestanden weder Wille noch Notwendigkeit zum Erhalt oder gar zum Ausbau der Mauer (LindesAy 2007).

natürliche Materialien genutzt wurden. Während in der Nähe von Touristenzentren einige Teile der Mauer erhalten oder sogar restauriert wurden, sind große Teile der Mauer heute in schlechtem $\mathrm{Zu}$ stand, wobei der schleichende Verfall insgesamt fortschreitet. Obwohl die Mauer bereits 1986 zum Weltkulturerbe erklärt wurde, wird sie erst seit 2006 in ihrem Bestand geschützt, was das Abtragen von Steinen zur Verwendung als Baumaterial oder gar den Abriss zur Schaffung von Raum für Bauvorhaben zumindest theoretisch verhindert.

\subsection{Die Mauer bei Peking}

In den Bergen nördlich von Peking verlaufen bis zu vier Mauern in Abständen von wenigen Kilometern parallel zueinander in Ost-West-Richtung.

Am Pekinger Knoten teilt sich die Mauer, die im Osten in Shanhaiguan (Pass zwischen Berg und Meer) ihren Anfang nimmt, in eine nördliche und südliche Linie. Während die südliche Linie im Westen bei Badaling und der massiven Festung am Juyong-Pass (der letzten Bastion $50 \mathrm{~km}$ vor Peking) nach Süden abbiegt und weiter bis nach Laiyuan führt, verläuft die nördliche Sektion ausgehend vom Pekinger Knoten über Zhangjiakou nach Nordwesten. Hinter Badaling weichen die Berge und umschließen eine Hochebene, die bei Jimingyi (Poststation zum Hahnenschrei) endet.

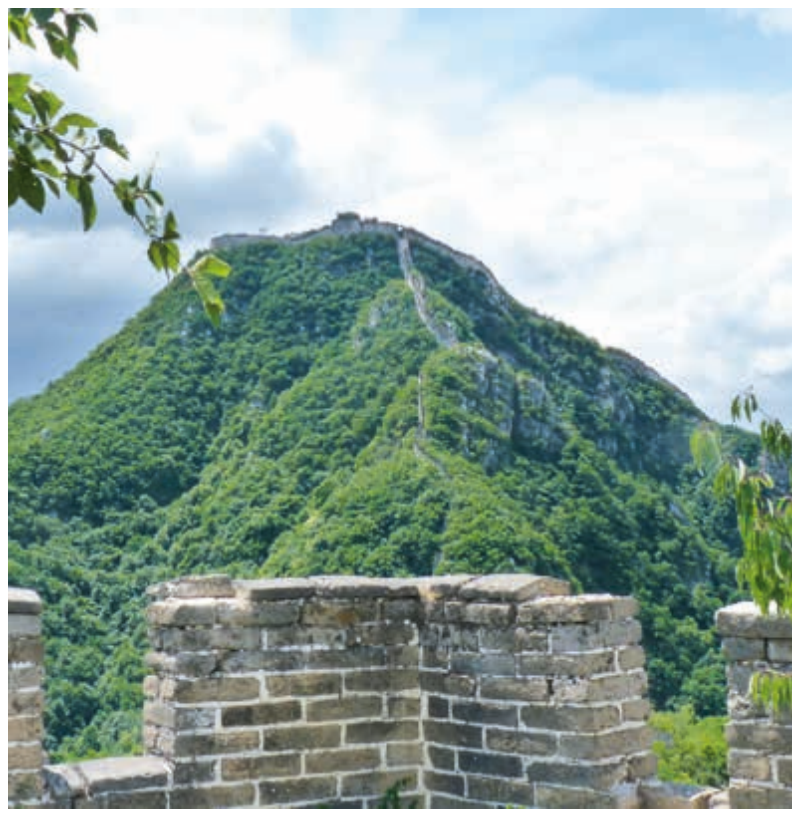

Abb. 4: Am Pekingknoten teilt sich die Mauer in eine nördliche und südliche Linie.

Der Weg von Peking (heute als Autobahn) über die Hochebene und den hinter Jimingyi liegenden Pass führt geradewegs in die Innere Mongolei nach Hohhot. Diese Hochebene von ca. 80 km Länge wird an ihrem Südrand durch eine zusätzliche Mauer in den Bergen und am Nordrand durch Wachtürme in der Ebene gesichert.

Im Gebiet von Peking misst die Mauer 4-8 m Breite auf der Krone und $10 \mathrm{~m}$ an der Basis, ihre Höhe schwankt zwischen 6 und $9 \mathrm{~m}$. Im Abstand von einigen hundert Metern befinden sich ungefähr $12 \mathrm{~m}$ hohe Türme, die als Waffenlager und Signaltürme dienten.

\subsection{Mauer von Jiankou}

Der Mauerabschnitt von Jiankou liegt etwa $70 \mathrm{~km}$ nordöstlich von Peking im Kreis Huairou und kann über eine seit 2005 ausgebaute Straße mit dem Auto inzwischen relativ schnell erreicht werden, obwohl man, um dorthin zu gelangen, zwei Pässe von um die $1200 \mathrm{~m}$ ü. d. M. überqueren muss.

Er umfasst ein ca. $20 \mathrm{~km}$ langes unrenoviertes Teilstück der Pekinger Verteidigungsanlagen, welches den touristisch erschlossenen Mauerabschnitt von Mutianyu im Osten mit dem von Huanghuacheng im Westen verbindet und verläuft in einem nach Westen ausschweifenden Bogen vom 


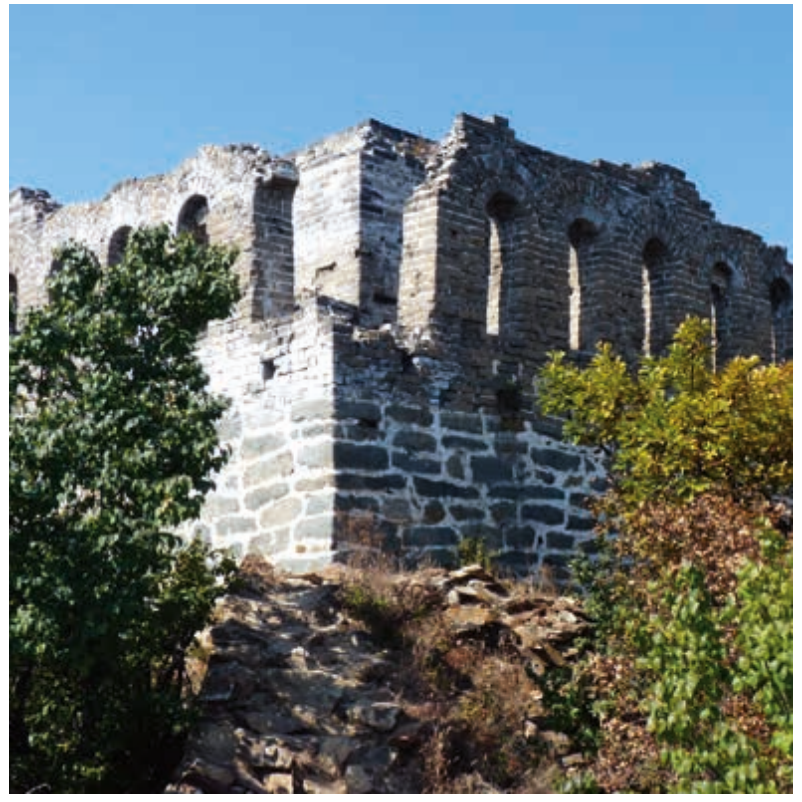

Abb. 5: Das Neun-Augen-Haus am Mauerabschnitt von Jiankou - einer der größten Mauertürme.

Niu Jiao Bian im Süden über den Pekinger Knoten zum Neunaugenhaus. Dies ist ein Mauerturm, der den Abschnitt von Jiankou überragt und seinen Namen von den auf jeder Turmseite befindlichen neun Fensteröffnungen herleitet. Dieser Turm ist einer der größten der chinesischen Mauer, gewöhnlich haben die Türme nur drei Fensteröffnungen auf jeder Seite.

Mit dem Bau des Jiankou-Abschnitts der Mauer wurde 1368 unmittelbar nach der Verlegung der Hauptstadt nach Peking begonnen. Die Errichtung sowie die Instandhaltung dieses Mauerstücks oblag den dort siedelnden Wehrbauern. Verwendet wurden für die Mauerbasis lokaler Dolomit sowie für die oberen Teile Ziegelsteine. Wie an vielen anderen Stellen im Gebiet von Peking gibt es auch in der Mauer von Jiankou kleine Durchlässe bzw. Pforten, die ein Überwechseln von einer Seite der Mauer auf die andere ermöglichen.

Der Mauerabschnitt von Jiankou verläuft über einen Bergkamm auf einer Meereshöhe zwischen 800 und $1200 \mathrm{~m}$, wobei zum Teil beträchtliche Höhenunterschiede bewältigt werden. Erst im

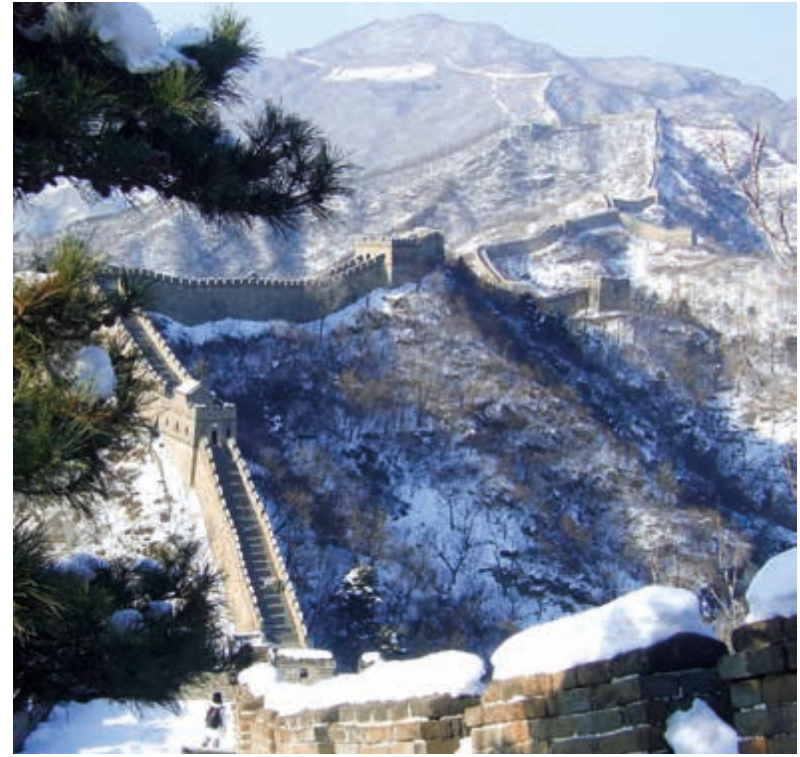

Abb. 6: Ein seltener Anblick: Schnee in den Pekinger Bergen, hier am Mauerabschnitt von Mutianyu südlich von Jiankou.

letzten Drittel verläuft die Mauer relativ flach auf etwa $900 \mathrm{~m}$. Dieser Bergkamm umschließt einen von Osten her zugänglichen Talkessel, in dem auf etwa $600 \mathrm{~m}$ das Dorf Xizhazi (westliches Gatter) liegt. Höchste Erhebung der Gegend ist der über dem Neunaugenhaus thronende Heituoshan mit $1534 \mathrm{~m}$.

\section{Klima}

\subsection{Peking}

Das durch kalte, frostige, schneearme Winter und heiße Sommer sowie durch extreme Trockenheit und große tageszeitliche Temperaturschwankungen gekennzeichnete Wüsten- und Steppenklima des Nordens reicht bis zu den Toren von Peking. Obwohl Peking nur etwa 150 Kilometer von der Küste entfernt liegt, wirkt sich das Seeklima in der Hauptstadt nicht aus, da diese im Westwindgürtel liegt. Auch sorgt der von Süden wehende Monsun insgesamt für ein Klima, das mit gemäßigt kontinental beschrieben wird. Der Jahresniederschlag beträgt $578 \mathrm{~mm}$ im Mittel, davon fallen etwa $62 \%$ in den Monaten Juli und August. Die Jahresdurchschnittstemperatur liegt bei $11,8{ }^{\circ} \mathrm{C}$. Der wärmste Monat ist der Juli mit einer Durchschnittstemperatur von $26^{\circ} \mathrm{C}$, der kälteste Monat ist Januar mit einer Durchschnittstemperatur von $-4,4^{\circ} \mathrm{C}$. 


\section{Vegetation}

\subsection{China}

Die Vielfalt der topographischen und klimatischen Verhältnisse in China führt naturgemäß zu einer großen Bandbreite an unterschiedlichen Lebensräumen und begünstigt damit auch die Ausbildung einer besonders artenreichen Flora. Die in China vorkommenden etwa 31000 Pflanzenarten, davon mehrere Tausend endemische, machen fast ein Achtel aller Pflanzenarten weltweit aus. Damit belegt China in der Liste der artenreichsten Länder den dritten Platz hinter Malaysia und Brasilien.

\subsection{Peking}

Die Gegend um Peking mit ihrem gemäßigten Klima befindet sich in der Vegetationszone der Laub-und Mischwälder. Diese bestehen überwiegend aus Pappel, Eiche, Kiefer, Lärche und Esche. Betrachtet man Fotografien von der Mauer z. B. bei Mutianyu oder Badaling, die um 1900 aufgenommen wurden, gewinnt man den Eindruck einer äußerst kargen Landschaft, auf der selbst das Gras nur dürftig wächst. Wenn überhaupt,

Die immer weiter um sich greifende Reduzierung der natürlichen Vegetation auf den nördlichen Gebirgszügen führte dazu, dass der Wald als natürlicher Schutzwall gegen die gefürchteten Sandstürme, die ihren Ursprung in den nordwestlich gelegenen Wüsten (namentlich der Wüste Gobi und der weiter im Westen gelegenen Wüste Taklamakan) haben, immer weniger seine Funktion als grüne Mauer ausüben konnte. Um einerseits eine Verringerung der Windgeschwindigkeit zu erreichen und damit die Auswirkungen der unvermeidlichen Sandstürme abzumildern und andererseits auch einer weiteren Desertifikation vorzubeugen, wurde 1979 das Projekt „Schutzwald in den drei Nordregionen " begonnen, in dessen Rahmen der Waldanteil im Norden Chinas von $5 \%$ (1977) auf $15 \%$ (2050) verdreifacht werden soll (zum Vergleich: der Waldanteil in Deutschland betrug 2012 rund $32 \%)$. sind auf den historischen Aufnahmen nur vereinzelt kleine Bäume oder Sträucher zu sehen. Als mögliche Gründe für diesen Zustand werden die Abholzung der Wälder aus militärischer Notwendigkeit bzw. zum Betreiben von Ziegelbrennöfen und häuslichen Kochstellen diskutiert. Sicher ist jedoch, dass mit der Industrialisierung Chinas ab den 1950er-Jahren und der damit einhergehenden intensiven Land- und Wassernutzung, die zu weiteren Abholzungen sowie zur Absenkung des Grundwasserspiegels geführt haben, sich die Standortvoraussetzungen für die natürliche Vegetation nicht verbessert haben.

Die Flora Pekings umfasst arktische Florenelemente, aufgrund des warmen kontinentalen Monsun-Klimas finden sich aber auch vereinzelt subtropische und tropische Pflanzen. Zudem sind Elemente der sibirischen Flora sowie einige eurasische Steppenpflanzen anzutreffen.

\subsection{Jiankou und seine „Mauerblümchen“}

Die Wanderungen der Autorin gingen immer vom Weiler Jiushuikeng aus. Vom Talkessel ging es nach Westen den Berg hinauf bis zur Mauerkrone, dann weiter in nördlicher Richtung auf der Mauerkrone dem Bergrücken folgend und schließlich bergab und über einen kleinen Sattel in östlicher Richtung zurück zum Weiler. Die Begehungen der Mauer wurden in meist wöchentlichen Abständen zwischen Mitte April und Mitte

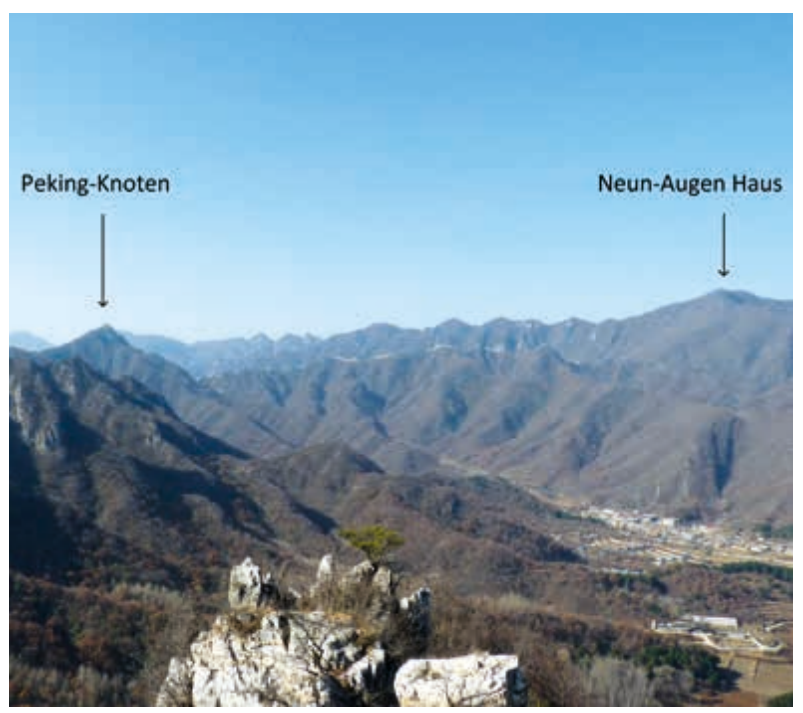

Abb. 7: Panorama mit Pekinger Knoten und Neun-AugenHaus. 


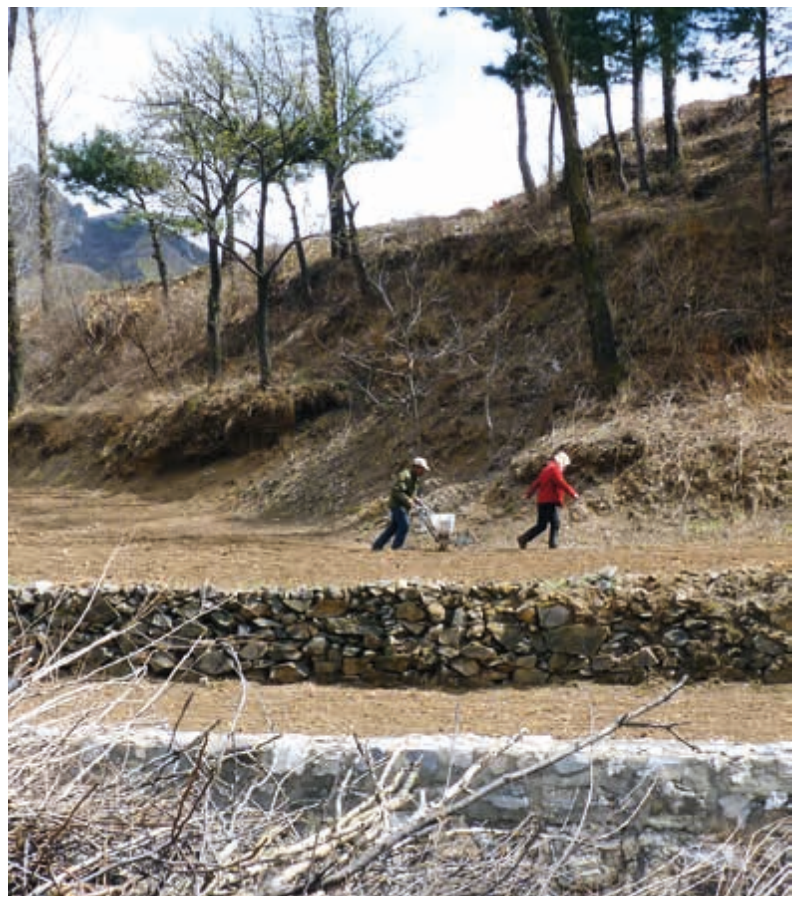

Abb. 8: Aufgrund der geringen Größe der Felder erfolgt Landwirtschaft überwiegend in Handarbeit.

Oktober 2011 sowie in etwas größeren zeitlichen Abständen in 2012 durchgeführt. So konnte die Vegetation in einem eng umgrenzten Gebiet an der Chinesischen Mauer fotografisch dokumentiert werden.

Die im Bereich des Mauerabschnittes von Jiankou wachsenden Pflanzen repräsentieren die im Pekinger Umland heimische Flora insgesamt recht gut. Dabei ist die vorhandene Vielfalt an Pflanzen, die im Laufe einer Vegetationsperiode auf der begangenen Tour zu finden sind, auch durch topografisch bedingte unterschiedliche Mikroklimata bedingt. Demzufolge sind manche Pflanzen nur an einer Stelle des Mauerabschnittes zu finden, andere hingegen sind durchgehend vorhanden.

Die Hänge oberhalb der Terrassenfelder um das Dorf Xizhazi sind mit Büschen und kleineren Bäumen bewachsen. Augenfällig ist im Frühjahr (Mitte April bis Anfang Mai) die Blüte des Chinesischen Berg-Pfirsichs (Prunus davidiana), die die Berghänge wie mit Watte überzogen aussehen lässt. Weiter hinten im Talkessel liegt Jiushuikeng. Auf dem dem Weiler zugewandten Schattenhang reichen die Azaleensträucher bis an die Bebauung heran, sie sind auch noch weiter oben als Unter-

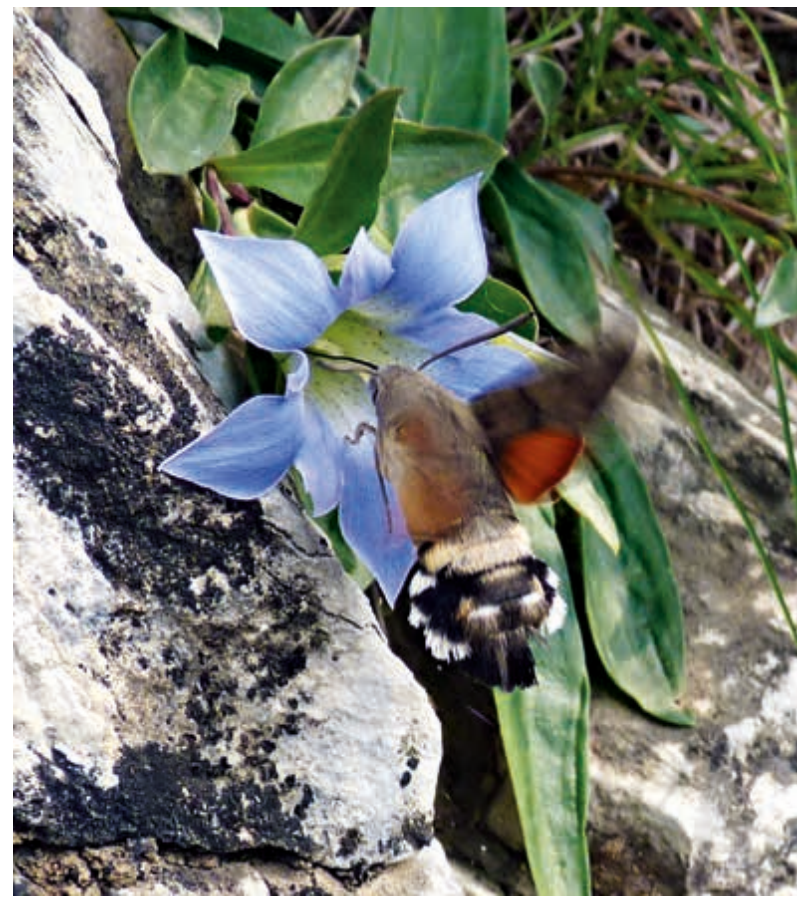

Abb. 9: Der Großblättrige Enzian (Gentiana macrophylla) wird von einem Taubenschwänzchen aufgesucht.

holz zu finden. Auf den Feldrainen oberhalb des Dorfes wachsen in der Nähe der Mauer:

Mehrere Veilchen-Arten (Viola prionantha, $V$. dissecta f. pubescens, $V$. acuminata), Mannsschild (Androsace umbellata), der Korbblütler Ixeris chinensis, Bergscharte (Rhaponticum uniflorum), Skabiose (Scabiosa tschiliensis), Tagblume (Commelina communis), Freiland-Gloxinie (Incarvillea sinensis), Lilie (Lilium concolor), Wiesen-Taglilie (Hemerocallis lilio-asphodelus), Edelweiß (Leontopodium smithianum), Großer Wiesenknopf (Sanguisorba officinalis) und Küchenschelle (Pulsatilla chinensis).

Auf dem Weg zur Mauerkrone folgen im Anschuss an die Felder Flächen, die bewachsen sind mit: Deutzien (Deutzia grandiflora und D. parviflora), Pfeifenstrauch (Philadelphus pekinensis), Spiere (Spiraea trilobata) und Weigelie (Weigelia florida).

Der Aufstieg zur Mauer erfolgt über einen kleinen Einschnitt in Ost-West-Richtung. Dieser Abschnitt liegt in einem Nordhang, daher ist es dort etwas feuchter. Dort befindet sich ein Pappelwald, außerdem wachsen dort Heckenkirsche (Lonicera elisae), Azalee (Rhododendron mucronu- 


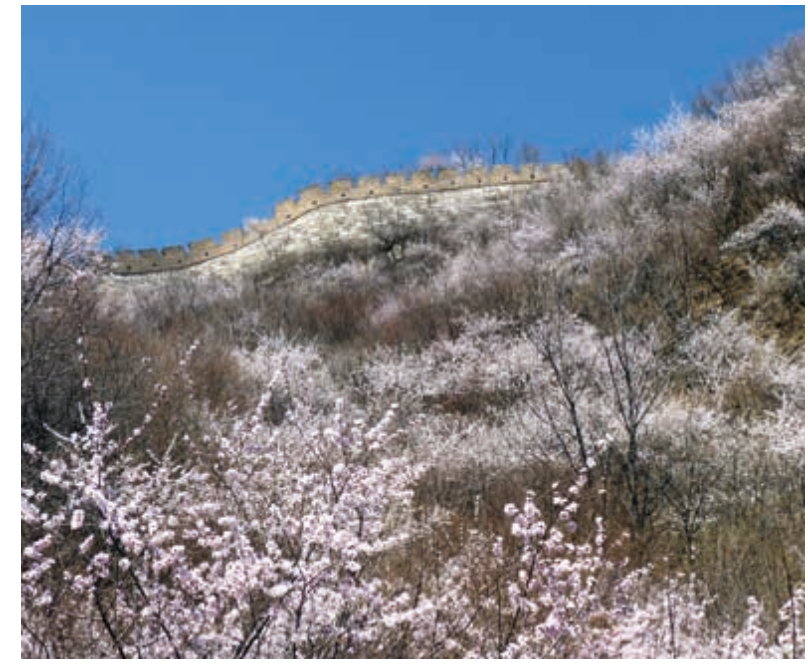

Abb. 10: Der Chinesische Berg-Pfirsich (Prunus davidiana) färbt im Frühling die Hänge weiß.

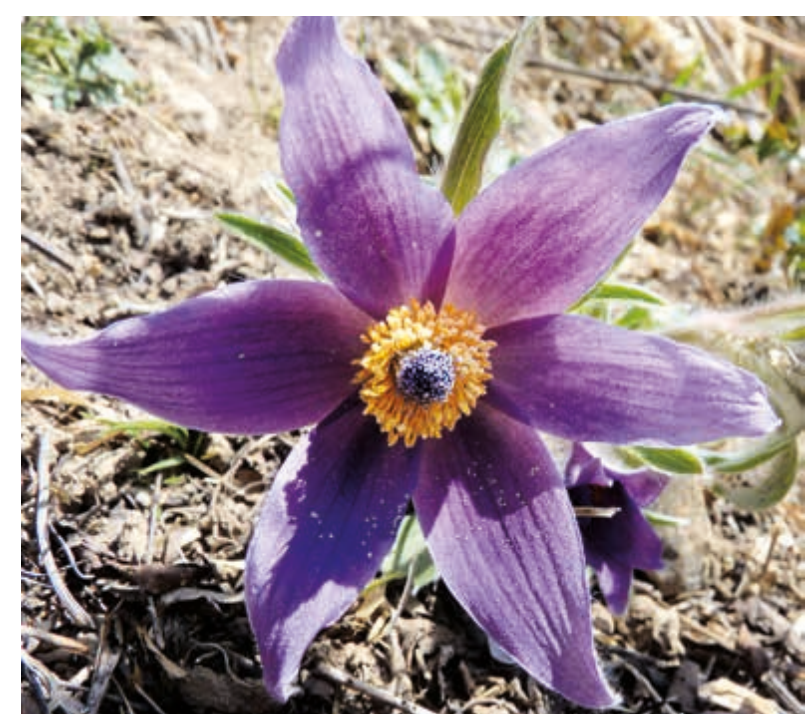

Abb. 11: Chinesische Küchenschelle (Pulsatilla chinensis).

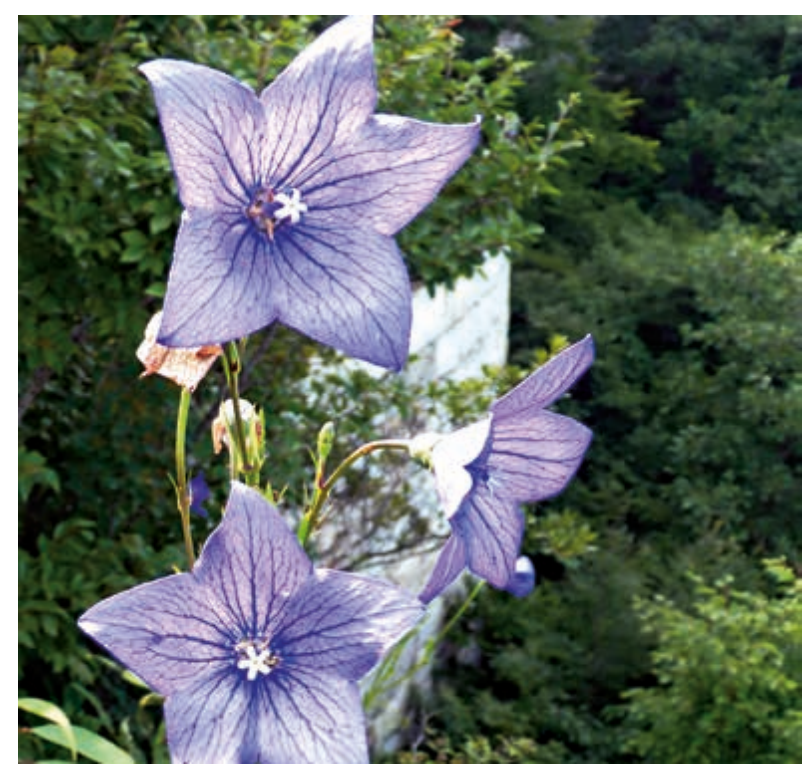

Abb. 12: Ballonblume (Platycodon grandiflorus). latum), Akelei (Aquilegia yabeana), Waldrebe (Clematis macropetala und C. brevicaudata), Eisenhut (Aconitum kusnezoffii) und die endemische Gerbera anandria. Oberhalb des Pappelwaldes steigt das Gelände hin zur Mauer nun steil an und liegt auch überwiegend in der Sonne. Hier wachsen die schon genannten Deutzien, Pfeifenstrauch (Philadelphus pekinensis), Spiere (Spiraea trilobata), Weigelie (Weigelia florida), auch einzelne Lärchen (Larix principis-rupprechtii), Schneeball (Viburnum sargentii) sowie vereinzelt auch Rhododendron (Rhododendron micranthum). Außerdem sind Erbsenstrauch (Lespedeza floribunda und $L$. bicolor), Maiglöckchen (Convallaria majalis), Läusekraut (Pedicularis tatarinowii), Waldrebe (Clematis brevicaudata), Esels-Wolfsmilch (Euphorbia esula), Spargel (Asparagus schoberioides), Labkraut (Galium spec.), Kreuzblümchen (Polygala sibirica), ein Malvengewächs (Pterospermum spec.) und Freiland-Gloxinie (Incarvillea sinensis) zu finden.

Am Fuß der Mauer und auf der Mauerkrone, die ohne nennenswerte Steigungen mehr oder weniger auf einer Höhe Richtung Norden verläuft, ist es sonnig und sehr trocken. Dort wachsen Chinesischer Berg-Pfirsich (Prunus davidiana), Sibirische Aprikose (Prunus sibirica), Mandschurische Kirsche (Prunus tomentosa), Chinesische Fontanesie (Fontanesia fortunei), Flieder (Syringa pubescens und $S$. reticulata var. amurensis), eine Gerbera-Verwandte (Myripnois dioica), Zweiblütige Abelie (Abelia biflora), Kleinblütiger Rhododendron (Rhododendron micranthum), Chinesischer Schneeflockenstrauch (Chionanthus retusus), Spiere (Spiraea trilobata), Spindelstrauch (Celastrus orbiculatus), Dünnblatt (Leptodermis oblonga), Fingerkraut (Potentilla spec.) und Ussuri-Scheinrebe (Ampelopsis glandulosa var. brevipedunculata). Außderdem finden sich in diesem Bereich Schwertlilien (Iris ruthenica und I. dichotoma), Korallen-Lilie (Lilium pumilum), Koreanischer Enzian (Gentiana pseudo-aquatica), Ballonblume (Platycodon grandiflorus), verschiedene weitere Glockenblumengewächse (u. a. Adenophora divaricata), Chrysanthemen (Dendranthema chanetii und D. lavandulifolia), Astern (Aster lauturianus, Aster ageratoides, Heteropappus altaicus, Doellingeria scaber), Baikal-Helmkraut (Scutellaria baica- 
lensis), Salomonssiegel (Polygonatum odoratum, P. humile und P. sibiricum), Lauch (Allium victorialis und A. macrostemon), Bergscharte (Rhaponticum uniflorum), Drüsige Kugeldistel (Echinops latifolius), Fetthenne (Sedum aizoon), Goldbaldrian (Patrinia spec.), Klebschwertel (Ixeris chinensis und I. sonchifolia), Kreuzblümchen (Polygala sibirica) sowie diverse Gräser.

Der Abstieg erfolgt über einen Einschnitt in West-Ost-Richtung, der sich zu einer kleinen Hochebene weitet; zum Teil sind die Hänge terrassiert, wohl um Erosion zu vermeiden. An dem Steilabstieg von der Mauer hinunter findet sich wieder vermehrt Chinesischer Berg-Pfirsich. Im Schatten der Bäume wachsen Waldhyazinthe (Platanthera chlorantha), Duftsiegel (Smilacina japonica) sowie, wie bereits beim Aufstieg, Läusekraut (Pedicularis tatarinowii) und Spargel (Asparagus schoberioides).

Weiter unten schließt sich eine kleine Ebene an, die locker bewachsen ist mit Weißdorn (Crataegus pinnatifida), Birne (Pyrus spec.), Erbsentrauch (Caragana rosea), Spindelstrauch (Celastrus orbiculatus) und Buschklee (Lespedeza floribunda). Auf den grasigen Flächen zwischen den Bäumen und Stauden finden sich Milchigweiße Schwertlilie (Iris lactea var. chinensis), Waldrebe (Clematis heracleifolia und $C$. hexapetala), Tauernblümchen (Lomatogonium carinthiaca), Kreuzblümchen (Polygala sibirica) und Großer Wiesenknopf (Sanguisorba officinalis).

Über einen kleinen Bergrücken, der mit Pappeln bewachsen ist, unter denen aber ganzjährig kaum blühende Pflanzen zu finden sind, geht es über einen felsigen, sonnigen Abhang hinunter zurück zum Dorf. Im oberen Teil des Abhangs wachsen wieder Fingerkraut (Potentilla spec.), Tauernblümchen (Lomatogonium carinthiaca), Weißer Eisenhut (Aconitum barbatum), Braune Akelei (Aquilegia viridiflora), Bergscharte (Rhaponticum uniflorum), Drüsige Kugeldistel (Echinops latifolius), Chrysantheme (Dendranthema lavandulifolia), Tagblume (Commelina communis), Chinesische Nelke (Dianthus chinensis) und kurz vor Erreichen

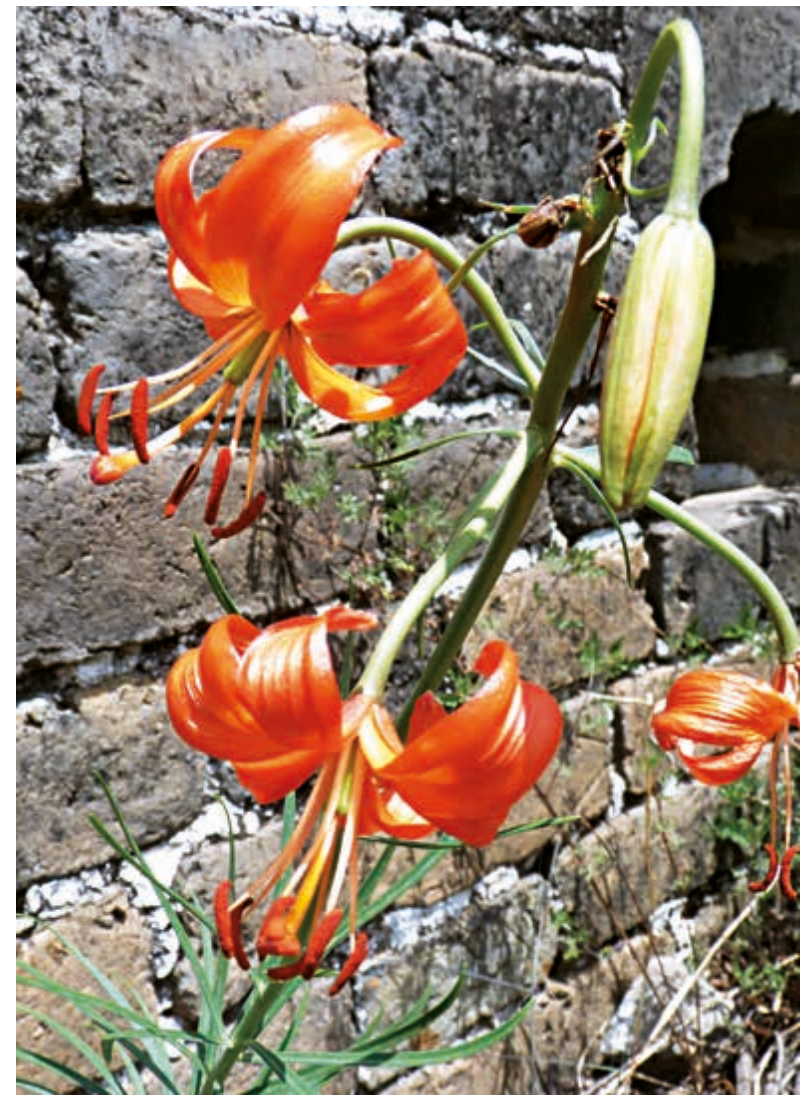

Abb. 13: Korallen-Lilie (Lilium pumilum).

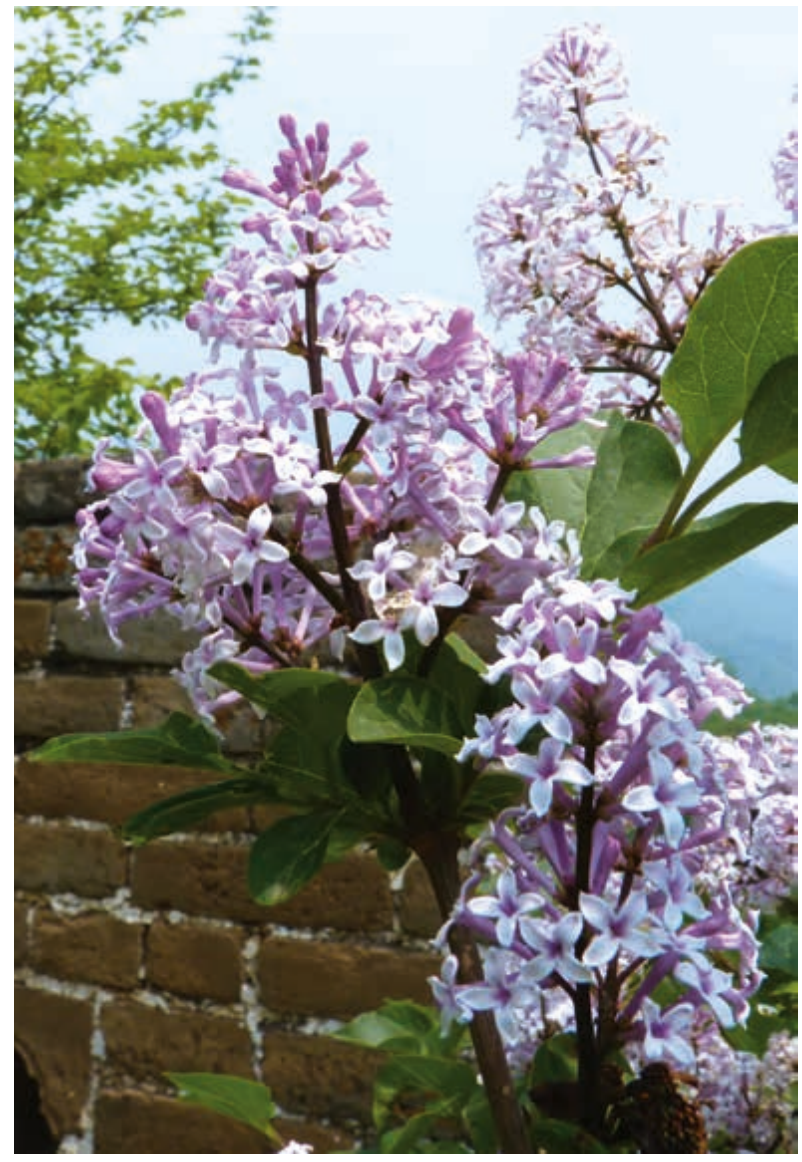

Abb. 14: Behaarter Flieder (Syringa pubescens). 
des Weilers einige Exemplare der Lampionblume (Physalis alkekengi).

Da die Fahrtzeit nach Jiankou auch von Peking aus bereits mindestens zwei Stunden beträgt, ist ein Besuch des Asien-Beetes im Palmengarten eine lohnenswerte Alternative. Dort sind neben einigen in diesem Artikel genannten Pflanzenarten zudem auch Pflanzen aus anderen Teilen Chinas zu sehen.
https://en.wikipedia.org/wiki/Jiankou

https://de.wikipedia.org/wiki/Volksrepublik_China

https://de.wikipedia.org/wiki/Region_(China)

https://de.wikipedia.org/wiki/Peking

https://de.wikipedia.org/wiki/Zhangjiakou

https://de.wikipedia.org/wiki/Klima_in_China

http://www.china-reisefuehrer.com/china-klima.html

http://www.hceis.com/ChinaBasic/Vegetation/Vegetation.

htm

https://de.wikipedia.org/wiki/

Chinas_Gr\%C3\%BCne_Mauer

\section{Literatur}

LindesAy, W. 2007: The great wall revisited - from the jade gate to old dragons's head. - Peking.

\section{Internetseiten}

http://flora.huh.harvard.edu/china/mss/plants.htm

The flora of China:

https://de.wikipedia.org/wiki/Yuan-Dynastie

https://de.wikipedia.org/wiki/Ming-Dynastie

https://de.wikipedia.org/wiki/Yongle

https://de.wikipedia.org/wiki/Chinesische_Mauer

http://www.chinesischemauer.net/

die-chinesische-mauer-heute/

https://www.travelchinaguide.com/china_great_wall/scene/ beijing/jiankou.htm

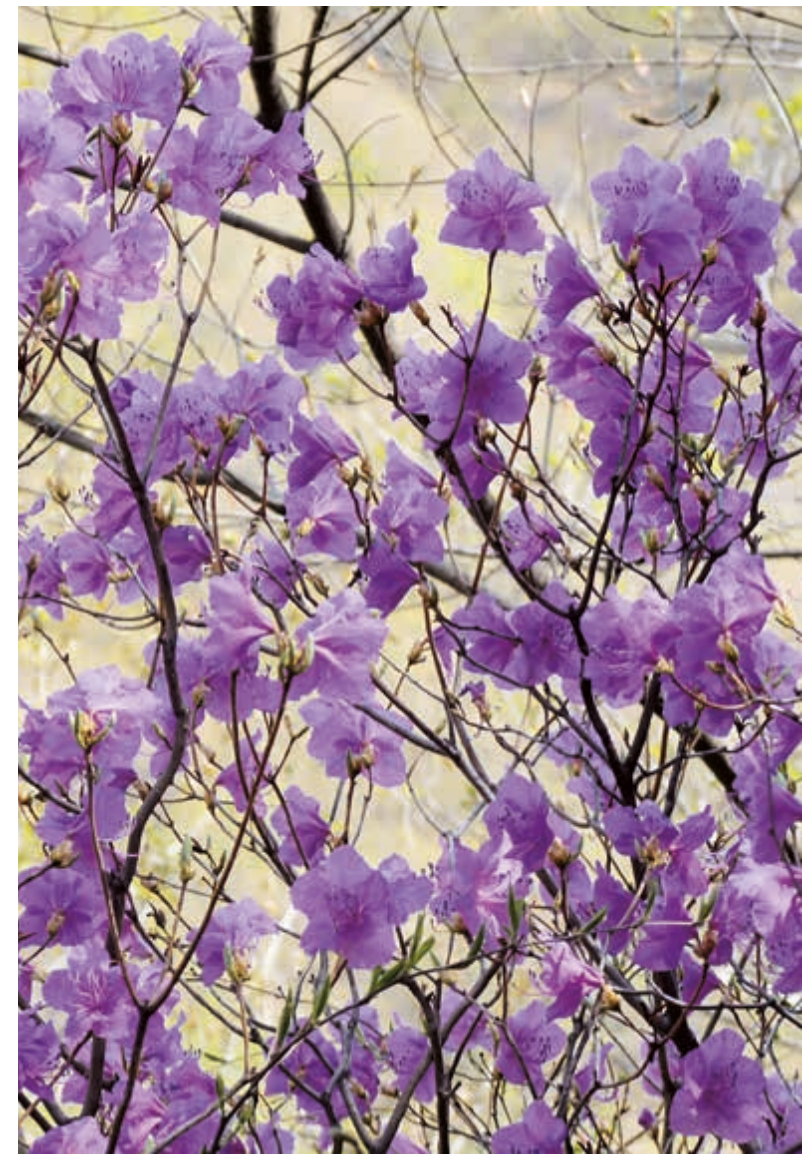

Abb. 15: Rhododendron (Rhododendron mucronulatum).

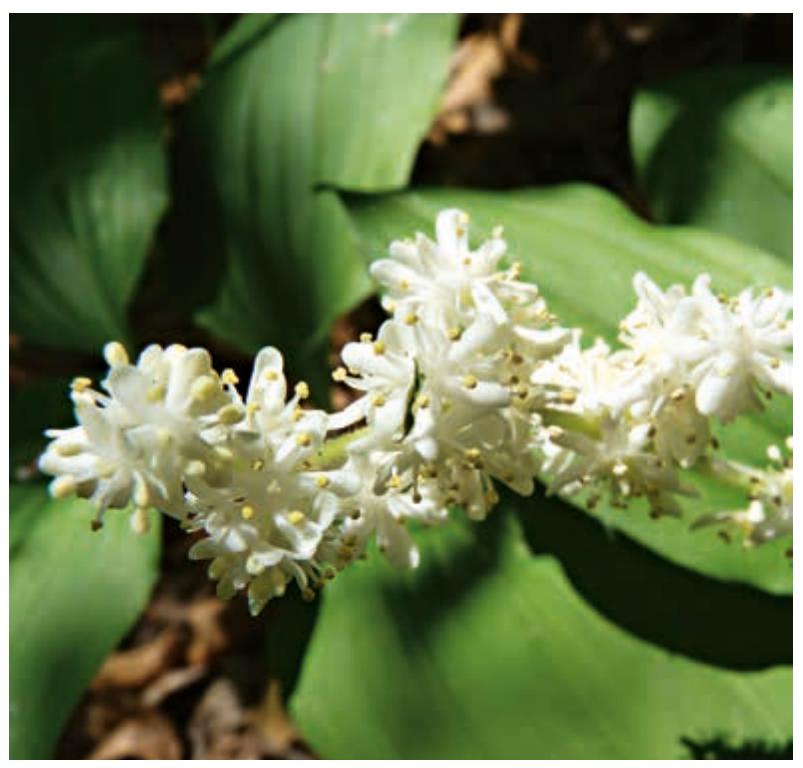

Abb. 16: Duftsiegel (Smilacina japonica).

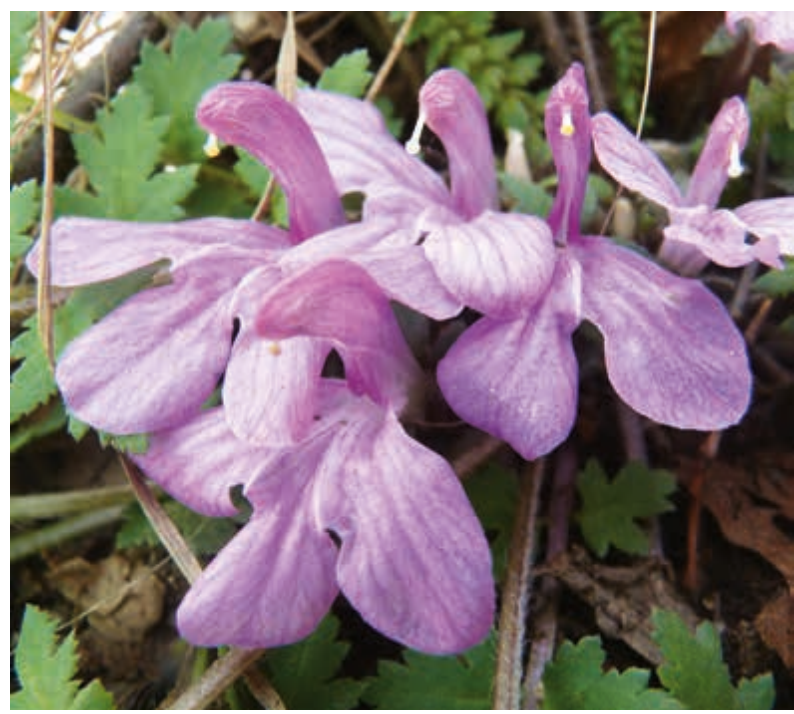

Abb. 17: Läusekraut (Pedicularis tatarinowii). 